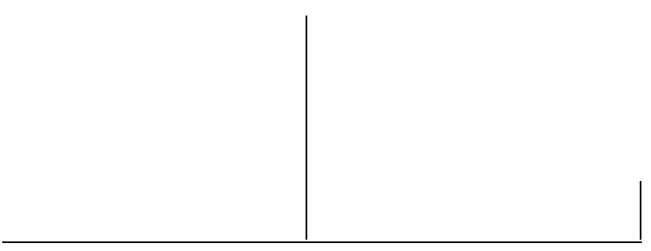

Rev. Latinoam. Psicopat. Fund., IX, 1, 5-17

\title{
Réflexions sur les fondements éthiques de l'acte clinique
}

Florence Gautier

Alain Ducousso-Lacaze

\begin{abstract}
Dans le domaine de la prise en charge psychothérapeutique la réflexion éthique est aujourd'hui reléguée à un second plan par le discours de l'efficacité et de l'évaluation. Or cette réflexion reste essentielle même si on peut considérer qu'elle a parfois été réduite à une rhétorique. Partant de la description d'un suivi psychothérapeutique référé à la psychanalyse, les auteurs proposent une démarche de recherche susceptible de mettre en évidence de manière rigoureuse la dimension éthique des actes cliniques. Dans une situation d'inceste la catégorie éthique de l'intime apparaît comme une valeur fondant l'ensemble de l'attitude du clinicien, ce qui interroge la validité d'une approche strictement évaluatrice et qui donc ne prendrait pas en compte cette dimension de l'acte clinique.
\end{abstract}

Mots clés: Intimité, inceste, acte clinique, éthique 


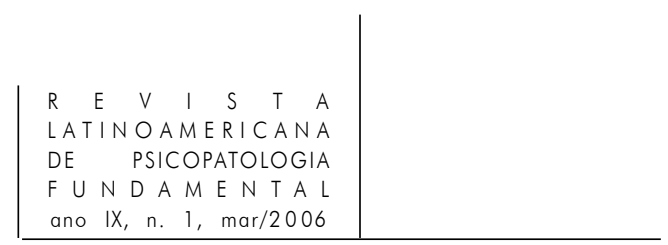

Etrange destin que celui de la réflexion éthique dans le domaine de la prise en charge à visée psychothérapeutique. Pour ceux qui se réfèrent à la psychanalyse, l'éthique a constitué pendant de nombreuses années le meilleur rempart contre l'accusation de manque de scientificité. Gori l'a fort bien dit: "L'éthique fut à la mode, sa rengaine nous consola de l'abandon de notre prétention à la scientificité" (Gori et Hoffmann, 1999). Dans ce contexte, seule la cure type semblait offrir les garanties nécessaires à la sauvegarde de la probité de chacun. En France nombreux furent ceux qui désertèrent les institutions de soin pour se replier sur le cadre psychanalytique (Gauchet, 1977).

Or, aujourd'hui, le paysage s'est nettement transformé. Aux accusations de manque de scientificité s'ajoute l'actuelle perte de la croyance dans les grands récits de la modernité et tout particulièrement dans le grand récit de l'émancipation. N'oublions pas que celui-ci légitimait de manière plus ou moins explicite la plupart des pratiques sociales centrées sur le sujet, la subjectivité, la subjectivation (Ducousso-Lacaze, 2001). Ainsi la référence à l'éthique a-t-elle dû céder la place sur le terrain des prises en charge à visée thérapeutique au discours technicien et évaluateur. Indépendamment de l'intérêt que peut présenter l'évaluation des différentes formes de "traitement psychique”, on ne peut s'empêcher de remarquer l'hégémonie actuelle du discours évaluateur au détriment de tout autre questionnement. Les réflexions théorique et clinique devraient s'effacer devant l'exigence de rendre compte de la pratique psychothérapeutique ou psychanalytique selon les canons actuels de l'évaluation. Seule cette dernière, supposée scientifique, serait en mesure de nous dire quelque chose de ce qui est devenu le but unique: l'efficacité. 


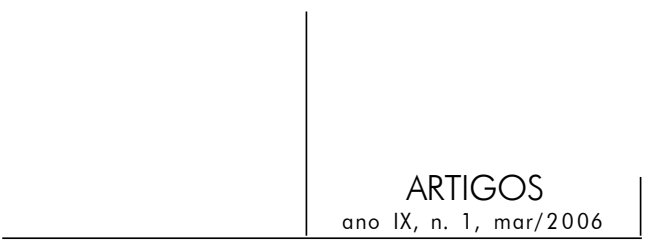

Le présent travail repose sur la conviction selon laquelle nous ne pouvons pas nous passer d'une réflexion éthique. Toutefois il nous paraît essentiel de ne pas retomber dans les ornières auxquelles nous avons fait allusion précédemment. A notre sens la réflexion éthique est à reprendre à partir de la clinique elle-même. Il s'agit en quelque sorte de montrer, données cliniques à l'appui, que les catégories éthiques ne relèvent pas de la seule rhétorique dans la confrontation des paradigmes, ni de la seule prise de position abstraite. Elles se trouvent au fondement de l'acte clinique lui-même (Gautier et Ducousso-Lacaze, 2004).

Afin de mettre en évidence l'intérêt d'une telle démarche nous procèderons en deux temps. Dans un premier nous rapporterons, de manière relativement classique, des données cliniques recueillies dans le cadre d'un travail psychothérapeutique réalisé auprès d'une enfant. Dans un second temps, nous proposerons une "relecture" de cette étude de cas à partir de concepts issus de certains travaux de Ricœur (1985) sur l'éthique. Notre objectif, dans ce travail, est limité. Nous souhaitons indiquer une voie de recherche possible pour la mise en évidence rigoureuse de la part éthique qui fonde les actes cliniques dans le travail psychothérapeutique.

Lise ou le droit à l'intimité ${ }^{1}$

Une passivité fondamentale

Lise, âgée de dix ans, présente une symptomatologie névrotique. En échec scolaire, agressive, inquiète, triste elle confie ses difficultés d'endormissement et prétend s'ennuyer vingt-quatre heures sur vingt-quatre. Dépourvue de discours spontané, à l'école elle est décrite comme une enfant discrète, effacée, qui présente de sérieuses difficultés de concentration. Les parents sont séparés dans un contexte de violence et de dispute. D'ailleurs Mme L. impute les problèmes de sa fille à cette séparation. Témoin de scènes d'agression qui l'amenaient à s'interposer; inquiète des menaces de suicides de sa mère et de son père, elle jouait auprès d'eux un rôle d'antidépresseur. Le divorce a stoppé cette situation délétère où la notion d'espace privé a maintes fois volé en éclat pour cette enfant. Finalement une psychothérapie s'amorce pour elle, à raison d'une fois par semaine.

Rapidement, Lise ressasse des récits qui tournent autour de moqueries, d'agressions physiques, d'insultes, dont elle est l'objet dans le cadre scolaire, et

1. Suivi psychothérapeutique réalisé par Florence Gautier. 


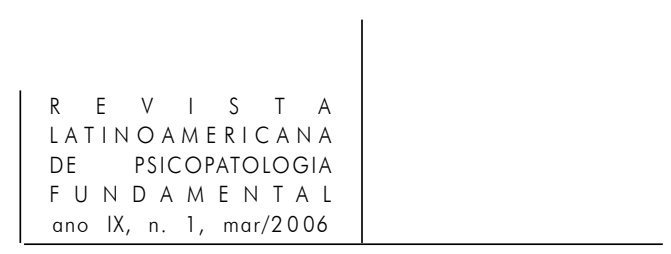

de sa position de bouc émissaire qui entraîne chez elle une réelle souffrance et tristesse. Sensible à la passivité fondamentale de Lise, j’opte sans détour pour lui interpréter symboliquement son comportement. Cette attitude clinique, soustendue par la pensée que quelque chose en elle attire le sadisme, constitue la variation essentielle effectuée auprès d'elle, afin de dénouer la satisfaction inconsciente de sa culpabilité.

Lise rase les murs du couloir du $\mathrm{CMPP}^{2}$ qui mène jusqu'à mon bureau. Lise a les pieds en dedans et quand elle court, elle se les emmêle et tombe. Lise reçoit, par ses "camarades", des coups de règles sur la tête pendant les cours. Lise, quelquefois, se venge, en douce, silencieusement. Cette enfant fortement névrosée, de structure hystérique, cherche en moi, en filigrane, une partenaire compréhensive. Elle demande le partage d'un vécu où elle est manifestement la victime. Elle me veut compatissante et consolante. Elle se plaint de cette situation et du caractère vain de ses défenses verbales, allant jusqu'à piquer des crises de nerfs qui ne la stigmatisent que davantage. Et toujours cela continue.

Lise s'offre, en quelques sortes, aux pulsions morbides d'autrui. Les autres la prennent pour objet: comme en réponse à sa demande inconsciente de punition. Cela a commencé au CP, me dit-elle. Un jour, elle se tient mal - adoptant alors une posture corporelle fatiguée, courbée, avachie qui continue de la poursuivre - et son instituteur la prévient que si elle continue ainsi, elle va devenir comme une mémé. Depuis, tout le monde l’appelle comme ça: “mémé!”. Ce signifiant qui s’est transmis de classe en classe la poursuit jusqu'à aujourd'hui, en CM2.

Cette tyrannie subie à l'école se prolonge dans sa famille où sa cousine s'allie à sa petite sœur, âgée de cinq ans, pour envahir sa chambre, lui casser ses jeux et l'exclure de leur amitié. Lise s'en plaint, revendique son droit à la paix. Mais l'autre jour encore elle s'est enfoncée dans le pied une punaise placée par sa petite sœur au bas de l'échelle de sa mezzanine. Elle n'a rien dit, sinon la petite fait des crises, se jette sur elle et la roue de coups.

Autant d'évocations où Lise est fondamentalement passive, en dépit d'une apparente rébellion; elle est absente de ses énoncés; elle est ailleurs. Autant d'opportunités de lui interpréter son inertie afin d'ébranler son statut de victime impuissante, de la sortir de l'au-delà mortifère dans lequel elle se cantonne: "Pourquoi c'est toi que l'on tape?”, “Tu supportes beaucoup de choses Lise”, “Tu ne vas pas te faire battre toute ta vie ?”, "Tu appelles ceux qui te 'tabassent' 'tes copains' et 'tes copines'?”

Comme elle mise sur le passage en sixième pour que cela cesse, je lui transmets la force de mon doute. Lorsqu'elle parle de "Buffy contre les 


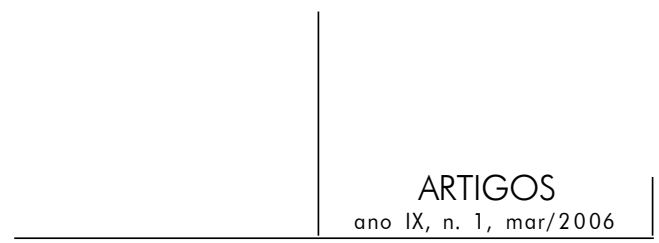

vampires", reconnue pour son courage à combattre les monstres, Lise veut me faire partager l'admiration qu'elle porte à cette jeune héroïne de série télévisée. Tout en me dégageant de sa recherche de complicité autour de cette idéalisation, la séance se clôt sur cette interprétation: “Et toi Lise qu'est-ce que tu combats?”

Quelques temps plus tard, Lise se tait, pendant plusieurs séances. Habillée d'un col roulé, elle relève cet habit jusqu’à son nez, bouche close, ferme et ouvre nerveusement son anorak. Elle se pince la peau sans répondre à mes sollicitations. Pendant trois semaines règne ce silence bruyant, rageur, destructeur.

Rentrée en sixième depuis trois mois, elle reprend le fil de ses mésaventures: les grands l'embêtent, la traitent de mouton parce que ses cheveux sont frisés, lui donnent des coups de pieds derrière son siège dans le bus. Sur le récit de cette misère, en surimpression, elle clame avec une énonciation inattendue, nouvelle, qui détonne du ton monocorde de ses plaintes: "Cette fois je ne me suis pas laissée faire. Je les ai engueulés. Même qu'ils soient plus grands, je m’en foutais!”

A la séance suivante elle ne vient pas, sa mère téléphone pour dire que la veille Lise a fait une crise de nerf et, finalement, a avoué subir une relation incestueuse de la part de son grand-père paternel depuis plusieurs années.

\section{Les effets pervers du souci de l'enfant}

Passé le temps de l’aveu, du récit à sa mère et auprès de la justice, Lise se trouve confrontée au discours maternel porteur de l’injonction de parler à la psychologue de la scène incestueuse: pour son bien-être; pour que ça aille mieux. Comme elle doit subir un examen gynécologique, Mme L. n’hésite pas à appeler plusieurs fois le CMPP pour faire part à la secrétaire de la situation, demandant à cette dernière qu'elle me transmette la nécessité de préparer sa fille à cette visite médicale. Mme L. expose par ses attitudes l'intimité de Lise. Elle ne protège pas son enfant par des limites symboliques qui instituent une sphère privée salutaire. Elle s'identifie à elle, sur un mode narcissique, et lui attribue ses propres inquiétudes. Lise n'a plus droit au secret: il faut qu'elle parle et pour son bien. Aulagnier (1976) nous rappelle que, pour le fonctionnement du Je, “une des conditions vitales est de pouvoir choisir les pensées qu’il transmet et celles qu’il garde secrètes” (p. 150).

Prenant en compte cette clinique qui transgresse l'intimité de cette enfant, je décide dans le cadre thérapeutique, de ne pas cristalliser dans le transfert de position d'attente surmoïque de récit et de pensée autour de la scène de l'inceste. Durant ses séances qui succèdent le temps de l'aveu, Lise expose une clef, qu'elle tient fermement entre ses mains. C'est la clef de son casier au collège, où elle met ses affaires “pour pas qu’on lui pique”. Elle l'exhibe, la dissimule et ainsi de suite. 


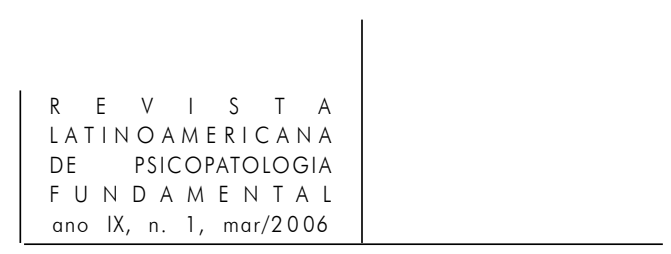

Cette clef, incessamment manipulée en thérapie, s’offre comme une matérialisation métonymique de l'accès à son intimité. Sur le mode de l'agir, Lise montre dans un malaise conflictuel qu'elle est maîtresse de cet accès.

Je reçois Mme L. en présence de sa fille pour une intervention symbolique décisive, symbolique car elle vise à instituer du tiers là où domine une relation duelle destructrice.

Elle relate l'agressivité de Lise qu'elle attribue aux attouchements du grandpère. En apprenant cet événement elle l'a couverte de cadeaux, et s'est montrée très permissive avec elle, pour la consoler. Elle s'imagine les pensées de sa fille autour de son grand-père, essayant de se mettre à sa place. Elle scrute sa fille du regard, tente de pénétrer son espace psychique, voudrait bien savoir ce qui se passe en elle. Elle raconte que suite à son refus qu'une copine de Lise dorme à la maison, sa fille s'est mise dans tous ses états et l'a tapée. Mme L. ne cesse de penser à l'inceste du grand-père paternel, et projette cette pensée obsédante sur sa fille. Il semble que la levée de l'interdit de l'inceste bouleverse le groupe familial dans son ensemble. D’ailleurs Mme L. agit de façon incestuelle (Racamier, 1997) avec sa fille, notamment en se plaignant de ses cachotteries. Dans une logique de séduction narcissique et d'emprise, elle voudrait être la copine, la confidente, en annulant toute règle de parenté, en excluant le tiers propre à l'ordre oedipien.

Le passage à l'acte de Lise sur sa mère prouve l'insupportable d'une situation traumatique où règne la confusion et l'abolition des espaces privés. J'interromps le récit de Mme L. en lui délivrant une parole qui vise avant tout à instaurer le droit à l'intimité pour Lise et à endiguer cette pensée pathogène et répétitive autour de la scène de l'inceste: “Mme L. ce qui devait être dit, a été dit. Ce qui devait être fait, a été fait. Vous avez porté plainte. Vous avez, auprès de Lise, tenu votre rôle de responsabilité parentale. Maintenant il faudrait peut-être arrêter de toujours parler de cet événement, de toujours y penser. En offrant des cadeaux à Lise, chacun de ces cadeaux vient le rappeler, et l'agressivité de votre enfant provient de cette situation. Il faut arrêter avec les cadeaux. Il y a vos pensées et celles de Lise qui sont différentes, et elle a le choix d'en parler ou de les garder pour elle. Vous dîtes qu'elle vous fait des cachotteries. Et là-dessus je peux vous répondre que c'est plutôt positif qu'elle s'autorise à ne pas tout dire à sa mère, qu'elle aie un espace pour elle. Heureusement, qu'elle ne vous dit pas tout."

Mme L. écoute et l'entretien s'achève sur cet acte de parole.

Cette position clinique spécifique se prolonge dans la psychothérapie où Lise est libre de décider de ce dont elle veut parler. C'est cet espace de subjectivation non orientée qui offre un déploiement à sa parole afin de reprendre sa vie dans le champ du plaisir: la curiosité intellectuelle, le goût de la lecture; la tenue d'un journal intime avec Laure; la mise en place de spectacles de danse avec sa cousine 


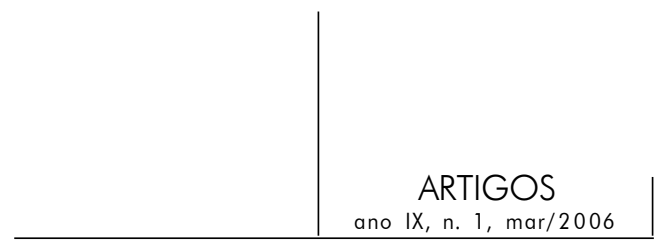

et ses amies; l'invention de musiques et de chansons à la flûte; la construction d'une cabane avec Charlotte, les détails de l'aménagement de cet habitat, le choix des personnes qui ont un droit d'entrée, selon des critères d’âge et de conduite respectueuse à tenir dans ces lieux privés envers les personnes et les objets...

Derrière ce récit métonymique d'un habitat s'entend la projection d'une construction d'un espace psychique réinvestit, un espace intime marqué de limites symboliques, revendicateur de son caractère privé et simultanément ouvert sur l'autre selon des règles définies (Vidal, 1999).

Lise émerge enfin comme sujet. Munie de sa flûte, elle me récite le refrain de la chanson qu'elle a inventé pour le spectacle avec sa cousine: "Je veux être moi, moi, moi, rien que moi!” Elle a cessé de se plaindre, au collège personne ne l'embête plus. En situation de réussite scolaire, elle atteste d'un véritable désir d'apprendre. Sa mère n'a plus avec elle cette attitude intrusive et un lien de filiation pacifiant transparaît au cours du récit des ballades familiales dans la forêt, où elles partagent le goût de la cueillette des champignons.

Elle décrit le sport à l’école: “J’aime faire la lutte, j’ai gagné deux matchs. Ma copine n’y arrive pas; moi si parce que je ne me laisse pas faire.”

Dans le cadre thérapeutique d’un espace de subjectivation non orienté, Lise s'est dégagée des rets mortifères, qui la retenaient sur la scène de l'inceste, lorsque cessèrent les intempestives demandes de mises en mots de sa réalité. Cette demande ravageante, soutenue par une logique de transparence et des vertus thérapeutiques du dire, faisait fi du droit à l’intimité, primordiale pour le sujet.

\section{Le pôle “tu” de l’intention éthique}

Tout au long des séances que nous venons de rapporter la psychologue a maintenu auprès de l'enfant une position spécifique. C'est elle que nous voudrions maintenant caractériser en partant de l'hypothèse que cette position, avant de relever d'une technique, trouve son fondement dans ce que Ricœur appelle "l'intention éthique".

Pour le philosophe, l'intention éthique repose sur un triangle de base comportant trois pôles: le pôle “je”, le pôle "tu”, le pôle "il”. Nous laisserons de côté le pôle “je” dans la mesure où il soulève la question de la liberté en première personne. A notre sens, l'ensemble de la séquence psychothérapeutique illustre avant tout le thème de la liberté en deuxième personne avec laquelle, pour Ricœur, on entre véritablement en éthique.

Sur le pôle “tu” se manifeste la volonté “que la liberté de l'autre soit”. Etrange formulation pour nous qui, habitués à la pensée psychanalytique, avons 


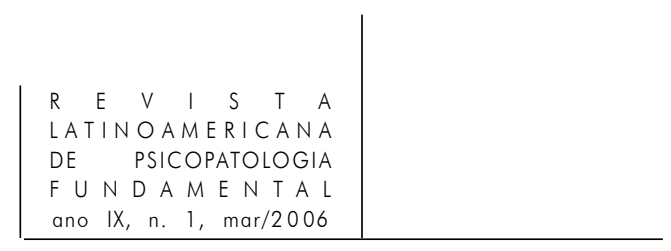

tendance à porter le soupçon sur ce vouloir pour l'autre. Certains d'ailleurs ont pu aller jusqu'à dire que le psychanalyste ne veut rien pour son analysant. Mais, est-ce si sûr? Peut-être s'agit-il avant tout de préciser ce qu'il faut entendre par cette formule: "Vouloir que la liberté de l'autre soit".

Elle désigne un acte que Ricœur qualifie d'acte de déliement dans la mesure où ce qui est en jeu c'est de rompre les liens qui enserrent l'autre. Or, dans la séquence psychothérapeutique que nous venons de rapporter, n'a-t-on pas affaire à des actes cliniques qui relèvent d'une telle intention éthique? Il nous semble que oui.

En effet, dans le premier temps de la psychothérapie, la psychologue, par les paroles qu'elle prononce, sollicite l'enfant d'une manière tout à fait spécifique. Elle tente en fait de le déloger d'une position psychique qui, selon elle, restreint considérablement la "liberté intérieure" de l'enfant. En ce sens on peut dire que la psychologue tente de lui permettre de se dégager de deux types de liens:

- les liens au sadisme de ses pairs;

- le lien à un déterminisme inconscient qui l'enferme dans une position subjective essentiellement passive.

Remarquons au passage la nuance que nous avons apportée par rapport aux propos de Ricœur. Plutôt que dire qu'il s'agit de rompre les liens qui enserrent l'enfant, nous avons préféré faire l'hypothèse que la psychologue tente de permettre à l'enfant de s'en dégager par elle-même. Cette nuance vient souligner une spécificité de l’intention éthique du psychothérapeute de formation psychanalytique: il cherche à créer les conditions d'une appropriation par le sujet lui-même de sa propre liberté.

Notons enfin l'affinité de ce que nous venons de dire avec la notion de processus de subjectivation qui est au cœur du travail de la psychologue. Cette notion désigne, rappelons-le, la possibilité, pour le sujet, de se situer dans un rapport singulier à ce qui le détermine. Elle pose donc que le sujet est en mesure de créer un écart par rapport aux représentations de lui-même qui l'assujettissent (Kaës, 1993). Le travail psychothérapeutique vise une reprise du processus de subjectivation qui, selon la psychologue et pour l'enfant considérée, se trouve dans une impasse. Or les commentaires que nous venons de proposer suggèrent qu'au fondement de cette visée se trouve une intention éthique dont la liberté de l'autre est l'enjeu.

\section{Le pôle "il” de l’intention éthique}

Mais la dimension éthique des actes cliniques de la psychologue n’est pas tout entière contenue dans ce que nous venons de dire. Il convient maintenant 


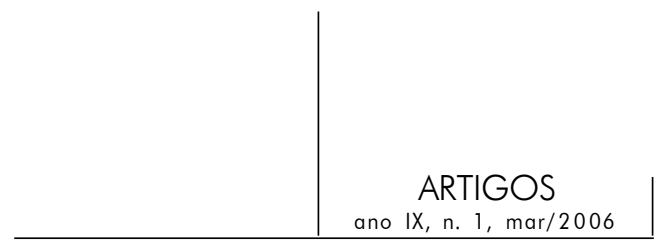

d'introduire le troisième pôle de l'intention éthique, le pôle “il”. Pour Ricœur, ce dernier permet de faire intervenir le tiers en tant qu'il constitue un référent commun. L'intention éthique articule un “je” et un "tu” en référence à un terme neutre, de la même manière que dans le langage toute relation dialogique exige un “il”, une chose placée entre deux sujets. Or le rôle de ce terme neutre c'est celui de la règle en ceci qu'il assure une médiation entre deux positions de liberté. Il s’agit donc de prendre en considération quelque chose comme une non personne qui, par son caractère abstrait, règle la relation intersubjective. C'est le monde des valeurs qui est cette fois sollicité, valeurs sédimentées dans la praxis collective et que tout sujet humain rencontre en s'éveillant à la conscience. Comment ce pôle “il” intervient-il dans la séquence psychothérapeutique que nous avons rapportée?

Selon nous il est à l’œuvre tout au long de cette séquence. Pour parler comme Ricœur nous dirons que la relation intersubjective est médiatisée par une valeur à laquelle se réfère aussi bien implicitement qu'explicitement la psychologue. Cette valeur nous l'appellerons la catégorie de l'intime. L'ensemble du processus psychothérapeutique nous paraît en effet guidé par l'exigence de faire advenir pour l'enfant un espace psychique qu'elle puisse reconnaître comme le lieu de son expérience intime à l'abri du désir et du regard des autres. La catégorie de l'intime en tant que valeur de référence soutient des attitudes et des actes cliniques qui attribuent une importance fondamentale, pour la liberté d'autrui et donc pour sa vie psychique, aux dimensions du secret et de la discrétion (Carel, 1992). Elle supporte, par exemple, l'ensemble du jeu symbolique qui se déploie autour de la clef du casier ainsi que le respect et l'attention que la psychologue manifeste à l'égard de ce jeu.

Soulignons au passage combien la notion d'aire intermédiaire de l'expérience est articulée à des considérations éthiques, ce qu'une certaine psychologisation affadissante conduit généralement à passer sous silence. On peut relire en ce sens toutes les remarques de Winnicott sur “l'attitude” de la mère qui permet l'émergence de cette zone intermédiaire dans la mesure même où elle s'abstient d'intervenir de manière intrusive dans le jeu de l'enfant soutenant de la sorte, ou participant à, l’illusion d'un trouvé-créé.

D’autre part la catégorie de l'intime fonde l'acte clinique décisif de la psychologue auprès de la mère de Lise, acte clinique sur lequel il convient de s’attarder. Nous insisterons sur deux points.

Tout d'abord, cet acte clinique met en évidence que la psychologue, dans son travail thérapeutique est amenée non seulement à se référer à des valeurs mais aussi à les traduire en acte. En effet, dans ce cas précis, les valeurs de référence de la psychologue s'opposent à celles de la mère de Lise. Celle-ci en effet rationalise son attitude en s'appuyant sur une valeur actuellement très en vogue: 


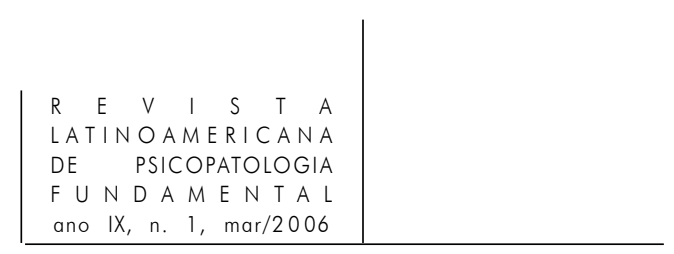

la transparence comme valeur ou l'exigence de tout dire. L'acte clinique en question, quant à lui, se fonde sur une défiance à l'égard de cette valeur. Dans un contexte où des actes incestueux ont été perpétrés, ce point prend un relief particulier. La révélation de l'inceste bouleverse toutes les catégories qui permettent de distinguer l'intime, le privé, le public. Elle apporte la publicité, nécessaire, à ce qui devrait rester de l'ordre du privé et de l'intime. Résister à l'exigence de transparence totale avec ses supposées vertus thérapeutiques, c'est, en l'occurrence, réintroduire l'intimité comme valeur susceptible de rétablir les catégories éthiques mises à mal.

D’autre part, cet acte clinique pose une question épineuse: celle du rapport du psychologue à la morale. Qu'entendons-nous par là? Suivant les réflexions de Ricœur nous envisagerons que le passage de l'éthique à la morale se fait avec le tournant de l'interdiction. Par son intermédiaire, s'opère une franche scission entre le préférable et le non préférable qui frappe le non préférable d’une valence négative. Or l'acte clinique en question dans sa structure même comporte une opération de ce type: il sépare le préférable du non préférable et projette l’ombre de l'interdiction sur ce dernier. En cela on peut dire qu'il glisse du champ de l'éthique proprement dite vers celui de la morale. Le psychologue peut-il aller jusque-là? Nous laisserons cette question ouverte mais nous ferons les remarques suivantes:

- Cet acte clinique ne porte pas sur les désirs ou les fantasmes de la mère de Lise mais uniquement sur ses conduites. De ce fait il opère, de manière implicite, une autre scission: il sépare les désirs ou les fantasmes de leur mise en acte. L'ombre de l'interdiction est donc projetée sur la dimension intrusive des conduites, et non sur les "pensées" de la mère de Lise.

- Il vise à faire advenir une autre séparation, entre la vie psychique de Lise et celle de sa mère. En cela on peut estimer qu'il constitue une actualisation de la fonction paternelle. Rappelons que si l'enfant a affaire au désir de la mère sans qu'une médiation s'actualise alors celui-ci est ouvert à "toutes les prises fantasmatiques”: il devient “l’objet de la mère” (Lacan, 1969, p. 373).

\section{Conclusion}

Parmi les critiques qui peuvent être adressées à notre travail, l'une d'entre elles pourrait consister à mettre en cause la conception de l'éthique qui nous sert de référence. Pourquoi celle-là? Pourquoi pas celle d'un psychanalyste?

A ces questions nous répondrons que penser l'éthique est tout autant nécessaire qu'extrêmement complexe. En ce domaine prétendre détenir la vérité 


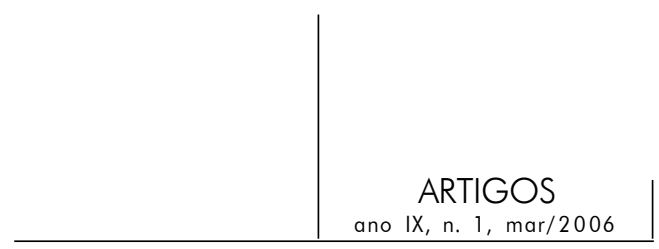

nous paraît tout à fait présomptueux, peut-être plus encore que dans d'autres domaines. Aussi la référence à la tentative de mise en forme de Ricœur ne saurait constituer pour nous un choix sans appel. En revanche elle présente, de notre point de vue, un double intérêt. D’une part elle permet de souligner la nécessité de faire un choix et de l'expliciter. Plus que le choix lui-même l'important est alors la démarche retenue qui consiste à confronter les faits cliniques à "une lecture" à partir de concepts spécialement forgés pour penser l’éthique. D’autre part, la référence à un philosophe nous oblige à nous décentrer par rapport à nos habitudes de penser et, peut-être, met plus clairement en évidence la part proprement éthique des actes cliniques comme des concepts que nous utilisons.

Pour terminer nous insisterons sur le fait que le cas clinique présenté n’a pas été choisi au hasard. De notre point de vue il illustre de manière particulièrement nette les risques que comporte l'hégémonie actuelle de la démarche évaluative. Comment rendre compte dans le langage de l'efficacité des enjeux éthiques fondamentaux que nous avons essayé de conceptualiser? La catégorie de l'intime peut-elle s’appréhender dans le cadre de grilles évaluatives? On peut d'ailleurs penser que la réduction de la réflexion au seul souci d'évaluation a pour fonction d'éviter de telles questions. Or dans son travail psychothérapeutique le psychologue n'est pas qu'un technicien. Le cas clinique présenté montre qu'il est parfois appelé à résister, pour des raisons éthiques, à certaines sirènes du discours social, spécialement du discours sur l'enfance, qui prônent tout à la fois la transparence, l’efficacité et l'évaluation. Il s’agit, en quelque sorte, de soustraire l'enfant à la "gourmandise” de l'adulte, l'adulte parent mais aussi parfois l'adulte technicien.

\section{Références}

Aulagnier, P. Le droit au secret: condition pour pouvoir penser. Nouvelle revue de psychanalyse, n. 14, p. 141-57, 1976.

Ducousso-Lacaze A. Nos psychothérapies sont-elles modernes? L'évolution psychiatrique, n. 66, p. 516-26, 2001.

Freud, S. (1920) Au-delà du principe de plaisir. In: Essais de psychanalyse. Paris: Payot, 1981.

Gauchet, M. De Pinel à Freud. In: Swain, G. Le sujet de la folie. Paris: Calmann-Lévy, 1977.

Gori, R. et Hoffmann, C. La science au risque de la psychanalyse. Paris: Érès, 1999.

KAËs, R. Le groupe et le sujet du groupe. Paris: Dunod, 1993. 


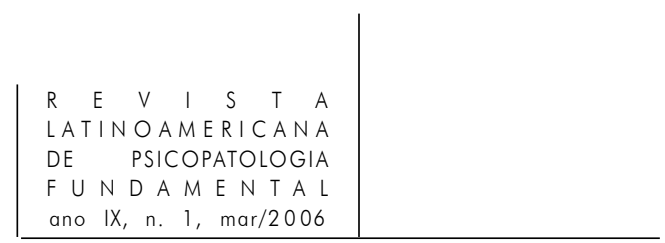

Filiation et affiliation. Le divan familial, n. 5, p. 61-78, 2000.

Lacan, J. (1969). Note sur l’enfant. In: Autres écrits. Paris: Seuil, 2001.

RACAMier, P-C. Brève histoire de l'incestuel. Groupal, n. 3, p. 7-16, 1997.

Ricéur, P. Avant la loi morale: l'éthique. In: Encyclopédia Universalis Les enjeux, 1985. p. 42-5.

VIDAL, J. P. L'habitat familial et ses rapports avec l'espace psychique. Le divan familial, n. 3, p. 13-30, 1999.

\section{Resumos}

No domínio do tratamento psicoterápico a reflexão ética é hoje relegada a um segundo plano pelo discurso da eficácia e da avaliação. Esta reflexão ainda é essencial mesmo quando é possível considerar que por vezes foi reduzida a uma retórica. Partindo da descrição de um acompanhamento psicoterápico com base psicanalítica, os autores propõem um procedimento de pesquisa capaz de evidenciar de modo rigoroso a dimensão ética dos atos clínicos. Em uma situação de incesto, a categoria ética do íntimo aparece como um valor básico do conjunto da atitude do clínico, o que questiona a validade de uma abordagem estritamente avaliativa e que, apesar de tudo, não consideraria esta dimensão do ato clínico.

Palavras-chave: Intimidade, incesto, ato clínico, ética

En el plano de las consideraciones a respecto da la psicoterapéutica, la reflexión ética es hoy relegada a segundo plano por el discurso de la eficacia y de la evaluación. Esta reflexión permanece esencial, a pesar de que a veces pueda ser reducida a una retórica. Partiendo de la descripción de un seguimiento psicoterapéutico de referencial psicoanalítico, los autores proponen un camino de investigación susceptible de evidenciar, de manera rigurosa, la dimensión ética de los actos clínicos. En una situación de incesto, la categoría ética de lo íntimo aparece como un valor que fundamenta el conjunto de la actitud del clínico, lo que interroga la validad de una aproximación estrictamente evaluadora y que no pretenda llevar en cuenta esa dimensión del acto clínico.

Palabras claves: Intimidad, incesto, acto clínico, ética

In the domain of psychotherapeutic treatment, any discussion of ethics today is given less importance than the discourse of effectiveness and evaluation. But a discussion of ethics is necessary when one realizes that it has at times been reduced to mere rhetoric. Based on the description of a psychoanalytically-based psychotherapeutic process, the author proposes a research procedure that is able to 


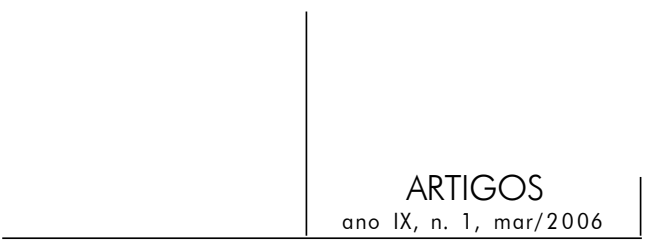

clearly highlight the ethical dimension of clinical acts. In a situation of incest, the category of the ethics of the intimate appears as a basic value in the overall attitude in clinical work. This questions the validity of a strictly evaluative approach which, in spite of everything, might fail to consider this dimension of the clinical act.

Key words: Intimacy, incest, clinical act, ethics 